\title{
Prefrontal Activity Links Nonoverlapping Events in Memory
}

\author{
Marieke R. Gilmartin, Hiroyuki Miyawaki, Fred J. Helmstetter, ${ }^{*}$ and Kamran Diba* \\ Department of Psychology, University of Wisconsin-Milwaukee, Milwaukee, Wisconsin 53211
}

The medial prefrontal cortex (mPFC) plays an important role in memory. By maintaining a working memory buffer, neurons in prelimbic (PL) mPFC may selectively contribute to learning associations between stimuli that are separated in time, as in trace fear conditioning (TFC). Until now, evidence for this bridging role was largely descriptive. Here we used optogenetics to silence neurons in the PL mPFC of rats during learning in TFC. Memory formation was prevented when $\mathrm{mPFC}$ was silenced specifically during the interval separating the cue and shock. Our results provide support for a working memory function for these cells and indicate that associating two noncontiguous stimuli requires bridging activity in PL mPFC.

\section{Introduction}

Learning to associate two events is more difficult when they are separated in time. For instance in fear conditioning, associating an auditory conditional stimulus (CS) and a shock unconditional stimulus (UCS) that occurs after an empty "trace interval" of several seconds requires multiple brain regions, including the medial prefrontal cortex (mPFC) (Runyan et al., 2004; Gilmartin and Helmstetter, 2010). Importantly, the mPFC is not required for learning if the cue and shock are contiguous (Morgan and LeDoux, 1995; Corcoran and Quirk, 2007; Gilmartin and Helmstetter, 2010). The mPFC has a well established role in working memory, and prefrontal neurons exhibit persistent firing during empty periods separating a cue and response (Fuster, 1973; Funahashi et al., 1989; Compte et al., 2003). Similar sustained firing has been observed in the prelimbic area (PL) of mPFC during the trace interval separating the CS and UCS in trace fear conditioning (TFC) (Baeg et al., 2001; Gilmartin and McEchron, 2005a), and blood oxygenation level-dependent signals obtained from functional magnetic resonance imaging are selectively increased in frontal cortical areas of humans during this interval (Knight et al., 2004; Haritha et al., 2013). These observations have led to the hypothesis that "bridging" activity in PL mPFC is necessary for the formation of fear memory. We recently showed that reversible inactivation of PL with muscimol or inhibition of PL NMDA receptors with APV before training impaired the formation of memory for TFC (Gilmartin and Helmstetter, 2010), providing further support for this hypothesis. However, these approaches

\footnotetext{
Received Jan. 11, 2013; revised May 23, 2013; accepted May 30, 2013

Author contributions: M.R.G., F.J.H., and K.D. designed research; M.R.G. and K.D. performed research;M.R.G. and H.M. analyzed data; M.R.G., F.J.H., and K.D. wrote the paper.

This work was supported by the National Institute of Mental Health Grant R01MH069558 (F.J.H.) and University of Wisconsin-Milwaukee Research Growth Initiative (K.D.). We thank D. Mueller for the use of his conditioning chamber and E. Stark for valuable suggestions. We would also like to thank E. Boyden, R.J. Samulski, and the University of North Carolina Vector Core for providing and preparing the viral vector.

The authors declare no competing financial interests.

*F.J.H. and K.D. contributed equally to this work.

Correspondence should be addressed to Marieke R. Gilmartin, Department of Psychology, University of Wisconsin-Milwaukee, 224 Garland Hall, 2441 East Hartford Avenue, Milwaukee, WI 53211. E-mail: marieke.gilmartin@gmail.com.

DOI:10.1523/JNEUROSCI.0144-13.2013

Copyright $\odot 2013$ the authors $\quad 0270-6474 / 13 / 3310910-05 \$ 15.00 / 0$
}

affect neuronal activity for minutes to hours and thus cannot test the role of $\mathrm{mPFC}$ activity specifically during the trace interval separating the cue and shock. Optogenetic tools allow us to limit the suppression of neuronal activity to the specific time hypothesized to be necessary for learning (Yizhar et al., 2011). Here we directly show that spiking activity in PL is required for the formation of fear memory by silencing PL neurons during the trace interval.

\section{Materials and Methods}

Subjects. The experiments were performed on 30 adult male Long-Evans rats (400-450 g; Harlan). All rats were housed individually and received food and water ad libitum. All procedures were in accordance with the National Institutes of Health guidelines and approved by the University of Wisconsin-Milwaukee Institutional Animal Care and Use Committee.

Stereotaxic virus injection and optic fiber implantation. Archaerhodopsin-T (CAG-ArchT-GFP) recombinant adeno-associated virus (AAV) was produced by Dr. Ed Boyden and serotyped (AAV9) and packaged by the University of North Carolina (UNC) Vector Core (titer: $2 \times 10^{12}$ molecules/ $\mathrm{ml}$ ). The map for AAV/CAG-ArchT-GFP is available on-line at http:// syntheticneurobiology.org/protocols/protocoldetail/39/10. The control virus AAV5/CAG-GFP (titer: $4 \times 10^{12}$ molecules $/ \mathrm{ml}$ ) was also obtained from the UNC Vector Core. Rats were anesthetized with isoflurane (induction, $4 \%$; maintenance, $1-2 \%$ ) and positioned in a stereotaxic frame. Using a $10 \mu \mathrm{l}$ syringe and 34 gauge needle (World Precision Instruments) mounted to a stereotaxic automated injector (InjectoMate; NeuroStar), $0.3 \mu \mathrm{l}$ of ArchT solution was slowly injected in PL mPFC (brain atlas target site: $15^{\circ}$ angle to vertical, anterior-posterior $+3.2 \mathrm{~mm}$; mediolateral: $1.6 \mathrm{~mm}$; dorsoventral $-3.7 \mathrm{~mm}$ from the skull) at a rate of $0.05 \mu \mathrm{l} / \mathrm{min}$. After the injection, the needle was left in place for an additional $10 \mathrm{~min}$ to allow diffusion away from the injector. This procedure was repeated for the opposite hemisphere. Immediately after injection of virus, fiber optic cannulae (Precision Fiber Products) were bilaterally implanted in PL $0.7 \mathrm{~mm}$ above the injection site. Each cannula assembly consisted of a $200 \mu \mathrm{m}$ diameter multimode Nufern fiber ( $0.22 \mathrm{NA}$ ) attached to a $2.5 \mathrm{~mm}$ diameter ceramic ferrule. Fibers were stripped and the tips were etched into a fine-tipped cone of $0.9 \mathrm{~mm}$ length with hydrofluoric acid. This fiber shape resulted in a broader cone of light (half-angle of $21-26^{\circ}$ ) emitted from the fiber tip, compared with a flat-cleaved fiber (half-angle of $9^{\circ}$ ) (Stark et al., 2012). This fiber shape helped to minimize tissue damage and affected a broader area of tissue in PL around the fiber tip. Using the on-line calculator of irradiance estimates through brain tissue (http://www.stanford.edu/group/dlab/cgi-bin/graph/chart. php) (Aravanis et al., 2007), the estimated irradiance at $1 \mathrm{~mm}$ from the fiber tip is $5 \mathrm{~mW} / \mathrm{mm}^{2}$ based on a half-angle of $21^{\circ}$ and $18 \mathrm{~mW}$ at the fiber tip $(\sim 2$ 
a

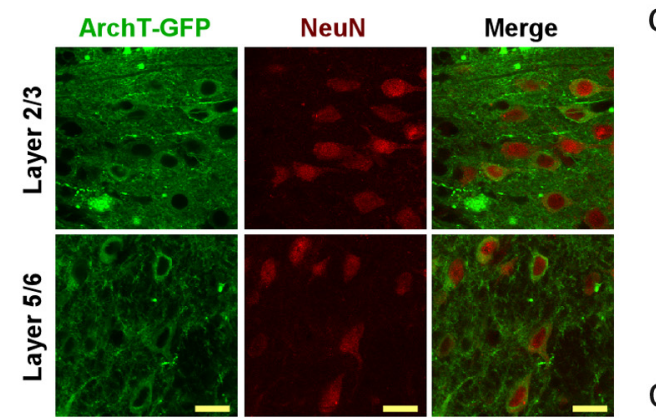

b

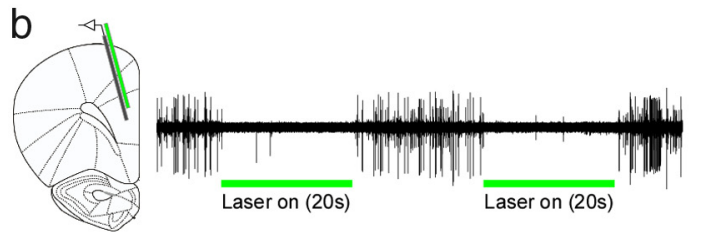

C

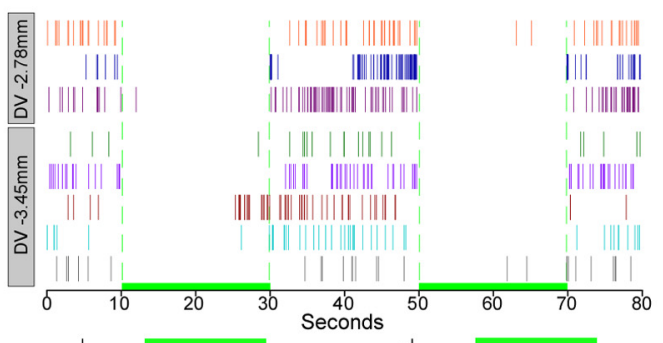

d

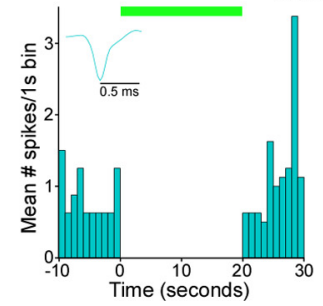

e

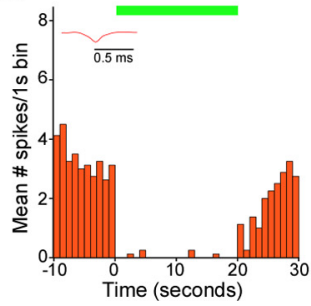

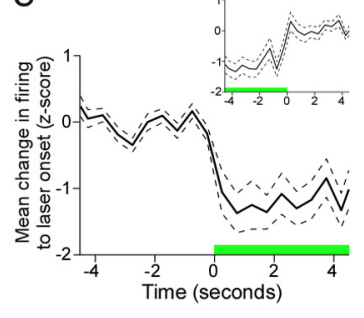

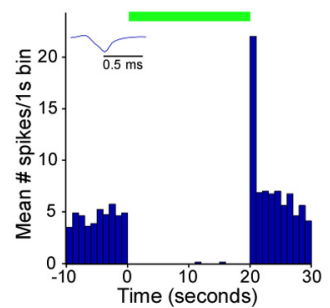

Figure 1. Laser illumination silences ArchT-expressing neurons in PL mPFC. $a$, Expression of AAV9/CAG-ArchT-GFP in layers $2 / 3$ and $5 / 6$ of PL and coexpression with the neuronal marker NeuN. Scale bar, 20 $\mu \mathrm{m} . \boldsymbol{b}$, Multi-unit activity recorded in PL showing population silencing during 20 s laser epochs in a rat recorded 3 weeks after virus injection $(532 \mathrm{~nm}, 12 \mathrm{~mW}$; green bar). $\boldsymbol{c}$, Action potential events of individual units recorded at two different depths in PL showing silencing during two 20 s laser presentations. $\boldsymbol{d}$, Peri-event time histograms of three representative units from $\boldsymbol{c}$ (same color) showing the change in firing to laser onset and offset. Each bar represents the number of spikes in $1 \mathrm{~s}$ bins averaged across eight 20 slaser presentations. The majority of units recorded did not show rebound activity at laser offset, but some units ( 1 of 7 units in $\boldsymbol{c}$, , right) exhibited brief rebound activity. $\boldsymbol{e}$, Normalized firing of all PL units recorded from the probe shank centered below the optic fiber $(n=59)$ across eight laser presentations showing the mean change in firing ( \pm SEM) to laser onset ( $g r e e n$ bar) relative to baseline. The inset shows the mean change in firing to laser offset.

a

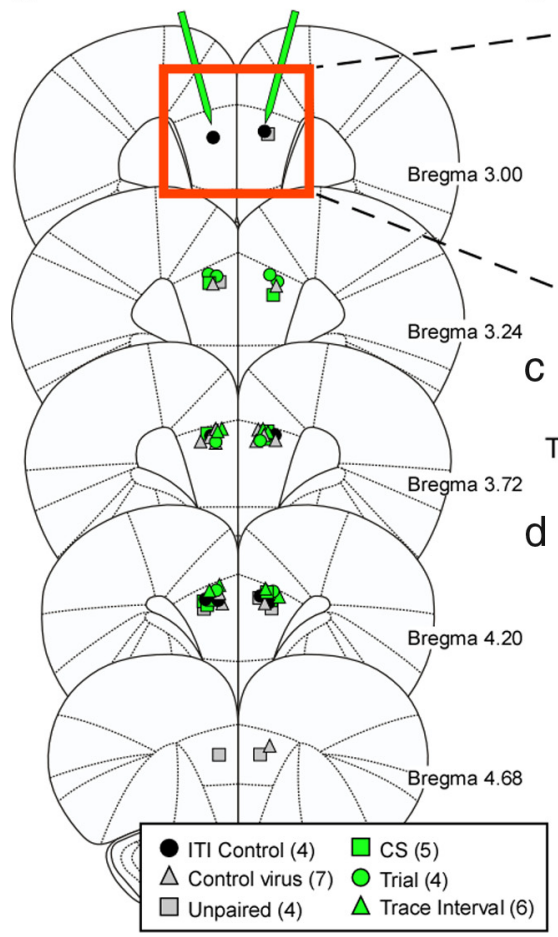

b

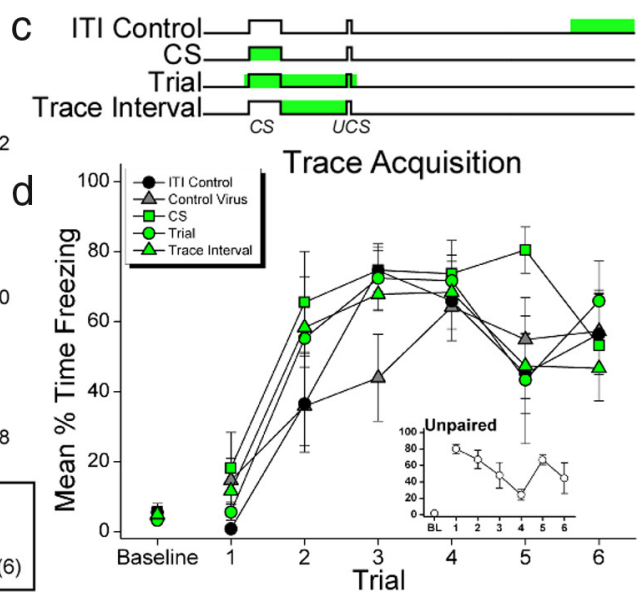

Figure 2. Temporally specific silencing of PL mPFC during conditioning. $\boldsymbol{a}$, Coronal diagrams show the placement of fiber tips in PL for each rat. $\boldsymbol{b}$, ArchT expression in PL mPFC and location of bilateral fiber cannulae in PL. c, Procedure diagram of light delivery (green bar) for each training group during conditioning. $\boldsymbol{d}$, Mean freezing during the training session of TFC. Each point shows the freezing during each trial (10s CS and $20 \mathrm{~s}$ trace interval). For the Trial, CS, and Trace Interval groups, the laser was on for all or part (CS group) of this period. The unpaired group received the first CS trial after two UCS trials. Freezing during each of the six CS trials is shown in the inset. All groups, including unpaired, showed equivalent freezing on the final trial $\left(F_{(5,24)}=0.321, p=0.895\right)$. Coronal diagrams were adapted from Paxinos and Watson, 2007, with permission from Elsevier.

$\mathrm{mm}$ from sensor). Ferrules were secured to the skull with skull screws, cyanoacetate, and acrylic cement.

Training and light delivery. Training was conducted 10-14 d after surgery in a Med Associates conditioning chamber (internal dimensions:
$30.5 \times 24.1 \times 29.2 \mathrm{~cm}$ ), housed in a soundattenuating outer chamber and illuminated with a white incandescent house lamp. Ventilation fans in each outer chamber provided 65-66 dB background noise and the white noise CS was delivered through a speaker in one side wall of the conditioning chamber. Stainless steel bars on the floor of the chamber served to deliver the footshock UCS. The chamber was cleaned with ethanol between rats. A single multimode patch cord with a fused optical coupler (Precision Fiber Products) split the light to two fibers for bilateral light delivery. This was attached to a collimated fiber port (Thorlabs) coupled to a green $(532 \mathrm{~nm})$ laser (UltraLasers). Light delivery was controlled with TTL pulses from Med Associates. One day before training, rats were acclimated to the patch cord attachment in the training chamber for $5 \mathrm{~min}$. On the day of training, rats were hooked up to the patch cords and placed in the chambers. After a 6 min baseline period, rats received six pairings of a $10 \mathrm{~s}$ white-noise CS $(72 \mathrm{~dB})$ and a $1 \mathrm{~s}$ footshock UCS (1 mA). The CS offset and UCS onset were separated by an empty $20 \mathrm{~s}$ trace interval and the intertrial interval (ITI) was $240 \pm 20 \mathrm{~s}$. Light was delivered to the PL at specific time points during training (see Fig. 2 for number of subjects/ group). In one group, light was delivered to the PL during the entire CS-UCS pairing on each trial, starting $1 \mathrm{~s}$ before CS onset and ending $1 \mathrm{~s}$ after UCS offset (Trial group). In a second group, light delivery was restricted to the $20 \mathrm{~s}$ trace interval (Trace Interval group). In a third group, light was delivered during the CS, either simultaneous with CS onset or $10 \mathrm{~s}$ before CS onset (CS group, data collapsed). In a fourth group, light was delivered for $20 \mathrm{~s}$ during the ITI (ITI Control group) at $60 \mathrm{~s}$ following the UCS to control for the temporal specificity of PL activity to learning the association. Two additional control groups were included. In one group, the rats were injected with a control virus lacking the 
ArchT proton-pump (AAV5/CAG-GFP) and light was delivered during the $20 \mathrm{~s}$ trace interval during trace conditioning (Control Virus group). In the second control group, the rats received unpaired control training, which consisted of the same number of CSs and UCSs as the paired groups, but the UCS and CS presentations were explicitly unpaired ( 12 trials, ITI $120 \pm 20$ s). This group served as a control for nonassociative responding to the CS. Light was delivered for $20 \mathrm{~s}$ during the ITI on 6 of the 12 unpaired trials, timed to match the number and timing of light presentations in the ITI Control group.

During paired training, rats learn to associate both the auditory CS and the training context with the shock UCS. The next day, rats were tested for memory of each association separately in the absence of any laser illumination. Conditional freezing to the CS was tested in a novel chamber in a separate room as previously described (Gilmartin et al., 2012). The CS retention test consisted of a 2 min baseline period followed by a brief $10 \mathrm{~s} C S$ and a long 5 min CS separated by 2 min (Gilmartin et al., 2012). This test allows us to assess freezing to both the CS and CS-offset. Contextual fear memory was assessed by measuring conditional freezing during 10 min re-exposure to the original training chamber.

Analyses. Freezing was defined as the cessation of all movement except that needed for respiration and was used as the measure of conditional fear during all training and testing sessions (Fanselow and Bolles, 1979). Freezing was scored automatically in real-time using the FreezeScan 1.0 detection software (Clever Sys). Statistical analyses were performed with Statistica, version 9 (Statsoft). Group differences in freezing were analyzed using one-way ANOVAs (context retention) or mixed model ANOVAs with repeated measures (acquisition; CS retention), which included the following factors: a repeated measure of Period (for acquisition: Baseline, Trials 1-6; for CS retention: Baseline, CS) and a between factor of Group. Only the first 2 min of the CS was analyzed to temporally match CS freezing with Baseline. Fisher least significant different post hoc tests were used to make pairwise comparisons on significant main effects and interactions. An $\alpha$ level of 0.05 was required for significance in all analyses.

In vivo neuronal recording. Three rats were injected with ArchT in PL $\mathrm{mPFC}$ as described above. Then 10,23 , or $36 \mathrm{~d}$ later, each rat was anesthetized with sodium pentobarbital $(50 \mathrm{mg} / \mathrm{ml}$ plus supplements as needed) and placed in a stereotaxic frame. An optrode was lowered into the right PL at a $15^{\circ}$ angle to vertical. The optrode consisted of a twoshank 16-channel silicon probe (NeuroNexus). Shanks were separated by $200 \mu \mathrm{m}$ and consisted of eight staggered recording sites (20 $\mu \mathrm{m}$ spacing). A single cleaved optic fiber was positioned $0.5 \mathrm{~mm}$ above one shank and secured to the electrode array (Stark et al., 2012). Neuronal activity was recorded at $30 \mathrm{kHz}$ with a DigitaLynx recording system (Neuralynx). During the recording sessions, laser light (532 nm, 8-12 mW) was delivered in epochs of $5 \mathrm{~s}, 10 \mathrm{~s}$, or $20 \mathrm{~s}$. The recorded data were median filtered (21-datapoint window) and subtracted from the unfiltered data. Individual units were extracted and sorted as described previously (Diba and Buzsáki, 2008) using open-source software (Hazan et al., 2006). Data were analyzed with MATLAB (MathWorks). Firing rates were calculated for each laser-on and laser-off period separately, and the resulting samples were compared with a Student's $t$ test. Units showing a significant $(p<0.05)$ decrease or increase in firing were classified as silenced or facilitated, respectively. Normalized firing ( $z$-scores; see Fig. $1 e$ ) during illumination was calculated for each unit relative to baseline $(10,500 \mathrm{~ms}$ bins in baseline and illumination periods).

Immunofluorescence and histology. At the end of the experiment, rats were deeply anesthetized with $5 \%$ isoflurane, transcardially perfused with 0.1 м PBS followed by $4 \%$ paraformaldehyde, and the brains were placed in fixative overnight. The following day, brains were transferred to a 30\% sucrose solution (in $0.1 \mathrm{~m}$ phosphate buffer) for cryoprotection. Brains were then frozen, sectioned coronally, and mounted on glass slides. Slides were coverslipped with anti-fade UltraCruz mounting medium (Santa Cruz Biotechnology). Images of GFP-expressing cells were obtained with a Nikon epifluorescence microscope and NIS Elements software (Nikon). For one rat, a subset of slices through the mPFC was set aside for immunofluorescence staining with the neuronal marker antiNeuN (Millipore). Slices were incubated in anti-NeuN (1:100) for $5 \mathrm{~h}$ at $4^{\circ} \mathrm{C}$ and in anti-mouse secondary conjugated to Alexa 594 for $1.5 \mathrm{~h}$. Slices were mounted and coverslipped with UltraCruz medium and imaged with an Olympus FluoView FV1200 confocal microscope $(60 \times$ oil objective).

\section{Results}

We injected rats bilaterally in PL mPFC with a viral vector containing ArchT and observed viral expression in the membrane and processes of PL neurons (Fig. 1a). To confirm functional membrane incorporation of the ArchT proton pump, we used an optrode consisting of a 16-channel silicon probe coupled with a $200 \mu \mathrm{m}$ core diameter fiber to simultaneously deliver light and record neuronal activity in three anesthetized rats $1-5$ weeks after viral infection. During illumination ( $12 \mathrm{~mW} ; 532 \mathrm{~nm})$ we observed robust silencing of spontaneous single-unit and multiunit activity (Fig. $1 b$ ) in each rat, suggesting a wide time window of functional ArchT for behavioral testing. This silencing was consistent across multiple illumination epochs (Fig. 1c,d). The firing during laser illumination was compared with prelight activity for each of the 112 units recorded from PL in this study. Fifty units (44.6\%) exhibited a significant decrease in activity during illumination compared with prelight activity. The majority of these units $(32 / 50)$ were recorded from the primary probe shank on which the fiber was centered. In addition to silencing, we observed 26 units (23\%) that exhibited an increase in firing during laser illumination. Almost all of these units (23/26) were recorded from the probe shank furthest from illumination, suggesting that a small set of units may be disinhibited during illu- 
mination of PL (Han et al., 2011). Figure 1e shows the normalized firing at laser onset for all units $(n=59)$ recorded from the primary probe shank.

We next tested whether PL mPFC spiking activity during the interval separating the CS and UCS is necessary for the formation of memory. Rats injected with ArchT or control virus were implanted with optic fibers bilaterally in PL (Fig. 2a,b). Following recovery from surgery, rats received six TFC trials. Light was delivered during the whole trial, CS, or trace interval to silence activity in these periods on each training trial (Fig. 2c). These groups were compared with a control group in which the PL was silenced during the ITI. To control for nonspecific effects of light delivery during the trace interval, a group of rats injected with a virus lacking the ArchT proton pump received light during the trace interval. Finally, an unpaired training group served as a nonassociative learning control (see Materials and Methods). Illumination of PL had no effect on the performance of freezing behavior during the training session in any of these groups (Fig. $2 d$; Group $\times$ Period: $\left.F_{(24,126)}=1.168 ; p=0.284\right)$. The following day, rats were tested for memory of the cue-shock association (Fig. 3). Groups in which light was delivered for the whole trial or just during the trace interval during training showed impaired memory for the auditory cue at test compared with control groups. This observation was supported by a Group $\times$ Period interaction, $\left.F_{(5,24)}=3.68, p=0.013\right)$. Both the Trial group and the Trace Interval group showed significantly less freezing during the CS compared with ITI controls $(p<0.015$ and $p<0.009$, respectively). CS freezing in these groups was not different from Unpaired controls $(p>0.05)$, demonstrating that silencing PL during the trace interval prevented the association of the CS and UCS. Importantly, illumination per se during the trace interval does not disrupt learning in nonArchT animals. The Control Virus group showed intact freezing equivalent to ITI Controls $(p>0.05)$ and significantly greater freezing than the Trial and Trace Interval groups ( $p<0.022$ and $p<0.012$, respectively). Silencing PL activity during the CS did not affect learning, as animals in this group exhibited intact freezing at test compared with controls $(p>0.05)$. This suggests that impaired freezing in the Trial group is due to silenced trace interval activity rather than disrupted CS firing. The memory impairment observed in the Trial and Trace Interval groups is specific to the CS-UCS association. All rats were able to associate the training chamber with the shock (Fig. $3 b ; F_{(5,24)}=2.27 p=0.08$ ), demonstrating that silencing PL during the trace interval did not interfere with the contextual association. These results indicate that the spiking activity of neurons in PL mPFC during the trace interval is critical for associating the CS with a shock that occurs several seconds later. Specifically, spiking activity in PL is required for the association of the auditory cue with the shock, but has no effect on learning to fear the training context, another prefrontal-dependent association (Gilmartin and Helmstetter, 2010). Thus, PL has a workingmemory function that is specific to the cue-shock association. Furthermore, dissociating cued from contextual learning in PL with selective silencing of bridging activity will allow us to identify the underlying neural mechanisms in prefrontal circuits supporting the context-shock association independent of cue-related neural plasticity.

\section{Discussion}

Our results provide important new information about the neural substrates of memory and the specific contributions of the mPFC. In fear conditioning, learning to fear a cue that predicts a shock depends on converging auditory and somatosensory in- puts to amygdala neurons with the mPFC serving a modulatory role on subsequent behavioral expression of fear (Romanski et al., 1993; Sotres-Bayon and Quirk, 2010). However, amygdala circuitry by itself cannot support learning when the auditory cue and shock are separated in time, and the mPFC is critically important (Gilmartin and Helmstetter, 2010; Guimarãis et al., 2011). The predicted role of mPFC in this case is to maintain a representation of the CS across the trace interval so that it can converge with shock-related neuronal activity. Cue-triggered persistent firing of prefrontal neurons has been observed in a number of learning paradigms, including trace conditioning (Fuster, 1973; Baeg et al., 2001; Gilmartin and McEchron, 2005a; Siegel et al., 2012), and could provide this bridging signal. Our findings directly support this possibility by demonstrating that silencing PL during the trace interval, but not during the CS or between trials, impairs memory. This temporal specificity of prefrontal activity was recently tested in another working memory task, delayed alternation. Using Channelrhodopsin in prefrontal interneurons, Yin et al. showed that disrupting delay period activity between a cue and response impaired performance of the task (Rossi et al., 2012). Our findings further suggest that PL activity during the cue preceding the trace interval or delay period is not necessary for trace conditioning. While PL units have been observed to exhibit increased firing to both the CS and trace interval during paired but not unpaired training (Gilmartin and McEchron, 2005a), we find that CS activity in PL is not critical to learning and that silencing PL does not affect the perception or salience of the CS. PL receives diverse input from cortical and subcortical regions and another structure, such as the hippocampus, likely provides the signal necessary to initiate bridging activity in PL. Not only is the hippocampus necessary for trace conditioning (McEchron et al., 1998; Czerniawski et al., 2012), but hippocampal units show learning-related activity during the CS (Gilmartin and McEchron, 2005b). Determining the functional significance of temporally specific input to PL on bridging activity and memory formation is now possible with optogenetic approaches. PL may provide a representation of the CS to the amygdala at the time the shock is delivered, allowing the two events to be associated. Alternatively, PL may coordinate persistent firing in rhinal cortices to bridge the gap (Egorov et al., 2002; Navaroli et al., 2012). Dysregulation of bridging activity in mPFC and its connection with fear circuitry may thus lead to inappropriate encoding and responding to threatening cues. More generally, cognitive processes requiring the association of nonoverlapping events would be sensitive to such dysregulation of mPFC. Age-related decline in episodic memory may be due in part to changes in prefrontal physiology that occur with age (McEchron et al., 2004; Kaczorowski et al., 2012). Our work clearly establishes PL firing during the interval separating two noncontiguous stimuli as a critical component of successful memory formation and advances our understanding of the role of $\mathrm{mPFC}$ in associative learning.

\section{References}

Aravanis AM, Wang LP, Zhang F, Meltzer LA, Mogri MZ, Schneider MB, Deisseroth K (2007) An optical neural interface: in vivo control of rodent motor cortex with integrated fiberoptic and optogenetic technology. J Neural Eng 4:S143-156. CrossRef Medline

Baeg EH, Kim YB, Jang J, Kim HT, Mook-Jung I, Jung MW (2001) Fast spiking and regular spiking neural correlates of fear conditioning in the medial prefrontal cortex of the rat. Cereb Cortex 11:441-451. CrossRef Medline

Compte A, Constantinidis C, Tegner J, Raghavachari S, Chafee MV, Goldman-Rakic PS, Wang XJ (2003) Temporally irregular mnemonic persistent activity in prefrontal neurons of monkeys during a delayed response task. J Neurophysiol 90:3441-3454. CrossRef Medline 
Corcoran KA, Quirk GJ (2007) Activity in prelimbic cortex is necessary for the expression of learned, but not innate, fears. J Neurosci 27:840-844. CrossRef Medline

Czerniawski J, Ree F, Chia C, Otto T (2012) Dorsal versus ventral hippocampal contributions to trace and contextual conditioning: differential effects of regionally selective nmda receptor antagonism on acquisition and expression. Hippocampus 22:1528-1539. CrossRef Medline

Diba K, Buzsáki G (2008) Hippocampal network dynamics constrain the time lag between pyramidal cells across modified environments. J Neurosci 28:13448-13456. CrossRef Medline

Egorov AV, Hamam BN, Fransén E, Hasselmo ME, Alonso AA (2002) Graded persistent activity in entorhinal cortex neurons. Nature 420: 173-178. CrossRef Medline

Fanselow MS, Bolles RC (1979) Naloxone and shock-elicited freezing in the rat. J Comp Physiol Psychol 93:736-744. CrossRef Medline

Funahashi S, Bruce CJ, Goldman-Rakic PS (1989) Mnemonic coding of visual space in the monkey's dorsolateral prefrontal cortex. J Neurophysiol 61:331-349. Medline

Fuster JM (1973) Unit activity in prefrontal cortex during delayed-response performance: neuronal correlates of transient memory. J Neurophysiol 36:61-78. Medline

Gilmartin MR, Helmstetter FJ (2010) Trace and contextual fear conditioning require neural activity and NMDA receptor-dependent transmission in the medial prefrontal cortex. Learn Mem 17:289-296. CrossRef Medline

Gilmartin MR, McEchron MD (2005a) Single neurons in the medial prefrontal cortex of the rat exhibit tonic and phasic coding during trace fear conditioning. Behav Neurosci 119:1496-1510. CrossRef Medline

Gilmartin MR, McEchron MD (2005b) Single neurons in the dentate gyrus and CA1 of the hippocampus exhibit inverse patterns of encoding during trace fear conditioning. Behav Neurosci 119:164-179. CrossRef Medline

Gilmartin MR, Kwapis JL, Helmstetter FJ (2012) Trace and contextual fear conditioning are impaired following unilateral microinjection of musci$\mathrm{mol}$ in the ventral hippocampus or amygdala, but not the medial prefrontal cortex. Neurobiol Learn Mem 97:452-464. CrossRef Medline

Guimarãis M, Gregório A, Cruz A, Guyon N, Moita MA (2011) Time determines the neural circuit underlying associative fear learning. Front Behav Neurosci 5:89. Medline

Han X, Chow BY, Zhou H, Klapoetke NC, Chuong A, Rajimehr R, Yang A, Baratta MV, Winkle J, Desimone R, Boyden ES (2011) A high-light sensitivity optical neural silencer: development and application to optogenetic control of non-human primate cortex. Front Syst Neurosci 5:18. Medline

Haritha AT, Wood KH, Ver Hoef LW, Knight DC (2013) Human trace fear conditioning: right-lateralized cortical activity supports trace-interval processes. Cogn Affect Behav Neurosci 13:225-237. CrossRef Medline

Hazan L, Zugaro M, Buzsáki G (2006) Klusters, NeuroScope, NDManager: a free software suite for neurophysiological data processing and visualization. J Neurosci Methods 155:207-216. CrossRef Medline

Kaczorowski CC, Davis SJ, Moyer JR Jr (2012) Aging redistributes medial prefrontal neuronal excitability and impedes extinction of trace fear conditioning. Neurobiol Aging 33:1744-1757. CrossRef Medline

Knight DC, Cheng DT, Smith CN, Stein EA, Helmstetter FJ (2004) Neural substrates mediating human delay and trace fear conditioning. J Neurosci 24:218-228. CrossRef Medline

McEchron MD, Bouwmeester H, Tseng W, Weiss C, Disterhoft JF (1998) Hippocampectomy disrupts auditory trace fear conditioning and contextual fear conditioning in the rat. Hippocampus 8:638-646. Medline

McEchron MD, Cheng AY, Gilmartin MR (2004) Trace fear conditioning is reduced in the aging rat. Neurobiol Learn Mem 82:71-76. CrossRef Medline

Morgan MA, LeDoux JE (1995) Differential contribution of dorsal and ventral medial prefrontal cortex to the acquisition and extinction of conditioned fear in rats. Behav Neurosci 109:681-688. CrossRef Medline

Navaroli VL, Zhao Y, Boguszewski P, Brown TH (2012) Muscarinic receptor activation enables persistent firing in pyramidal neurons from superficial layers of dorsal perirhinal cortex. Hippocampus 22:1392-1404. CrossRef Medline

Paxinos G, Watson C (2007) The rat brain in stereotaxic coordinates. Amsterdam: Academic

Romanski LM, Clugnet MC, Bordi F, LeDoux JE (1993) Somatosensory and auditory convergence in the lateral nucleus of the amygdala. Behav Neurosci 107:444-450. CrossRef Medline

Rossi MA, Hayrapetyan VY, Maimon B, Mak K, Je HS, Yin HH (2012) Prefrontal cortical mechanisms underlying delayed alternation in mice. J Neurophysiol 108:1211-1222. CrossRef Medline

Runyan JD, Moore AN, Dash PK (2004) A role for prefrontal cortex in memory storage for trace fear conditioning. J Neurosci 24:1288-1295. CrossRef Medline

Siegel JJ, Kalmbach B, Chitwood RA, Mauk MD (2012) Persistent activity in a cortical-to-subcortical circuit: bridging the temporal gap in trace eyelid conditioning. J Neurophysiol 107:50-64. CrossRef Medline

Sotres-Bayon F, Quirk GJ (2010) Prefrontal control of fear: more than just extinction. Curr Opin Neurobiol 20:231-235. CrossRef Medline

Stark E, Koos T, Buzsáki G (2012) Diode probes for spatiotemporal optical control of multiple neurons in freely moving animals. J Neurophysiol 108:349-363. CrossRef Medline

Yizhar O, Fenno LE, Davidson TJ, Mogri M, Deisseroth K (2011) Optogenetics in neural systems. Neuron 71:9-34. CrossRef Medline 